\title{
CalMagNet - an array of search coil magnetometers monitoring ultra low frequency activity in California
}

\author{
J. Cutler ${ }^{1,2}$, J. Bortnik ${ }^{3,2}$, C. Dunson ${ }^{2}$, J. Doering ${ }^{2}$, and T. Bleier ${ }^{2}$ \\ ${ }^{1}$ Space and Systems Development Laboratory, Department of Aeronautics and Astronautics, 496 Lomita Mall, Stanford \\ University, Stanford, California, USA \\ ${ }^{3}$ Department of Atmospheric and Oceanic Sciences, Room 7115, Math Sciences Building, UC Los Angeles, California, \\ 90095-1565, USA \\ ${ }^{2}$ Quakefinder, LLC, 250 Cambridge Avenue, Palo Alto, California, 94306, USA
}

Received: 19 September 2007 - Revised: 6 December 2007 - Accepted: 6 December 2007 - Published: 15 April 2008

\begin{abstract}
The California Magnetometer Network (CalMagNet) consists of sixty-eight triaxial search-coil magnetometer systems measuring Ultra Low Frequency (ULF), 0.001$16 \mathrm{~Hz}$, magnetic field fluctuations in California. CalMagNet provides data for comprehensive multi-point measurements of specific events in the Pc 1-Pc 5 range at mid-latitudes as well as a systematic, long-term study of ULF signals in active fault regions in California. Typical events include geomagnetic micropulsations and spectral resonant structures associated with the ionospheric Alfvén resonator. This paper provides a technical overview of the CalMagNet sensors and data processing systems. The network is composed of ten reference stations and fifty-eight local monitoring stations. The primary instruments at each site are three orthogonal induction coil magnetometers. A geophone monitors local site vibration. The systems are designed for future sensor expansion and include resources for monitoring four additional channels. Data is currently sampled at 32 samples per second with a 24-bit converter and time tagged with a GPS-based timing system. Several examples of representative magnetic fluctuations and signals as measured by the array are given.
\end{abstract}

\section{Introduction}

A number of magnetometer networks are deployed throughout the world to measure magnetic field variations to provide insight into the two-dimensional geographic distribution and dynamic variation of current flow and particle precipitation in the magnetospheric-ionospheric system.

Correspondence to: J. W. Cutler

(jwc@stanford.edu)
For example, the CANOPUS array deployed in northern Canada contains thirteen fluxgate magnetometers (along with additional riometers, photometers, and imaging systems) to monitor high-latitude ionospheric currents and auroral activity (Rostoker et al., 1995). The CANOPUS array is currently being expanded to include an additional 15 triaxial fluxgate magnetometers and eight two-axis induction coil magnetometers (Mann et al., 2004).

Another example, the Finnish pulsation magnetometer chain (Hebden et al., 2005), monitors geomagnetic pulsations with seven induction coil systems in Finland and one in Crete.

Magnetometer arrays are also used to probe conductivity structures of the Earth. Geomagnetic depth sounding (GDS) techniques use triaxial magnetic measurements to obtain orientations to nearby conductivity anomalies (Gregori and Lanzerotti, 1980; Schmucker, 1985). Typical GDS measurement examples include the survey of northern Italy by Armadillo et al. (2001) and a survey of North America by Neal et al. (2000).

Complementing the GDS technique which relies solely on magnetic field measurements, are magnetotelluric methods which use three axis magnetic and two axis electric measurements to determine the depth-profile of conductivity structures (Simpson and Bahr, 2005).

For example, a magnetotelluric network has been deployed as part of the Parkfield earthquake prediction experiment (Bakun and Lindh, 1985; Roeloffs and Langbein, 1994) to monitor sections of the San Andreas fault in California. UC-Berkeley has deployed two sites (Morrison et al., 1996), and an additional three sites are under development by Stanford University for deployment in the San Francisco Bay Area (Bijoor et al., 2005).

Published by Copernicus Publications on behalf of the European Geosciences Union. 


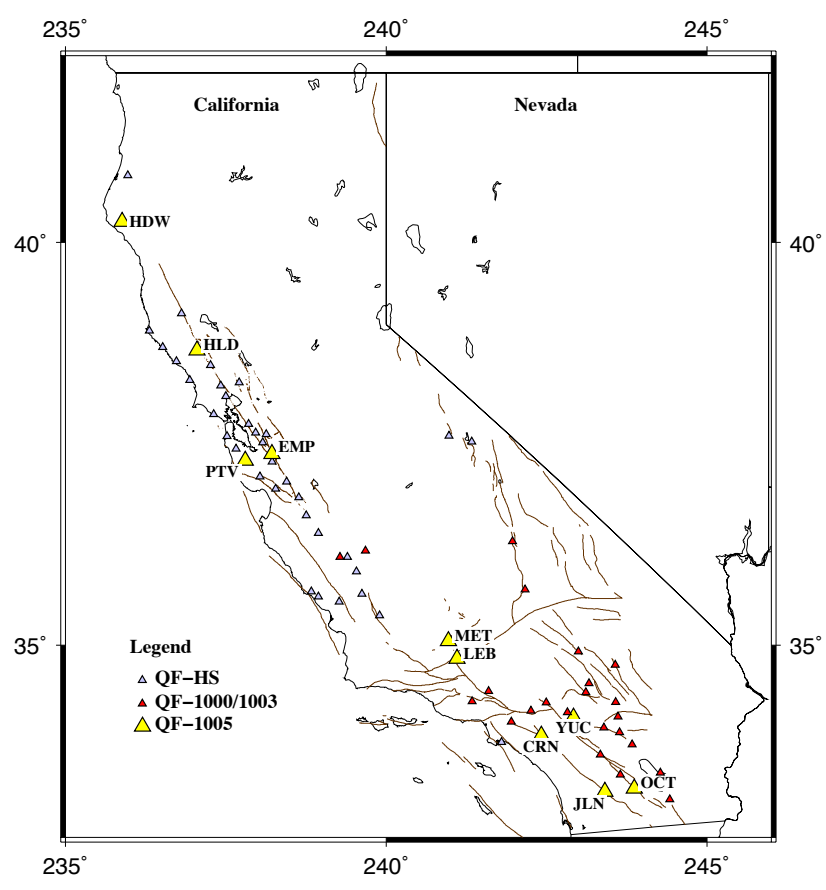

Fig. 1. A map of sensor locations in CalMagNet. The QF-1005 reference stations are shown as yellow triangles and labeled with station codes. Light blue and red triangles represent QF-HS and QF1000/1003 stations respectively. Station locations coarsely reflect the San Andreas and adjacent fault systems, and are placed in high probability earthquake regions.

To provide additional low-latitude magnetic field measurements, we have developed and deployed the California Magnetometer Network (CalMagNet), an array of sixty-eight induction coil magnetometer systems.

L-shell values, which approximately represent the number of Earth radii that the local magnetic field line extends into space (Campbell, 2003), of the CalMagNet sensor systems range from 1.6-1.9.

Example signals include geomagnetic micropulsations (Jacobs, 1970; Bortnik et al., 2007) and spectral resonant structures (SRS) associated with the ionospheric Alfvén resonator (Belyaev et al., 1990).

CalMagNet is also monitoring ULF activity in active fault regions of California. Reports have appeared in the literature indicating anomalous electromagnetic activity preceding large quakes (e.g. see reviews by Hayakawa (1999), Park et al. (1993), Johnston (1997) and the references therein).

In direct relevance to this paper, we note two observations made during large recent California earthquakes.

Fraser-Smith et al. (1990) and Bernardi et al. (1991) describe an anomalous signal preceding the the 18 October 1989 M7.1 earthquake that occurred in Loma Prieta, California. However, Park et al. (2007) detected no anoma- lous activity with electric dipoles preceeding the M 6.0 earthquake at Parkfied, California, on 28 September 2004.

Therefore, CalMagNet is being used for a systematic survey of ULF activity in diverse fault regions to assess any potential correlations between various earthquake events and ULF signals.

In this paper, we describe the CalMagNet sensor systems.

In Sect. 2, we describe our network topology and strategy for sensor placement.

In Sect. 3, details of the sensor systems are given, including a system block diagram, analog to digital conversion parameters, and sensor transfer function and noise floors.

In Sect. 4, we give examples of measured ULF signals and events.

We conclude in Sect. 5 with a summary of system capabilities and data distribution methods.

\section{Network topology}

Site selection for CalMagNet sensors is primarily directed by our long-term strategic goal to provide sensitive measurements of magnetic field fluctuations in the ULF range, located as close as possible to all land-based earthquakes greater than magnitude 5 in California. With a desired maximum distance of $10 \mathrm{~km}$ from an epicenter, this would require over 100 sensor systems along the over $2000 \mathrm{~km}$ of active fault zones in California. Due to cost constraints, this is not currently feasible.

Therefore, we guide our network topology and sensor placement with statistical methods indicating potentially higher probability locations for large earthquakes. Areas of increased seismic potential are identified from the output of a technique for describing driven, nonlinear threshold systems, the Phase Dynamics Probability Change (PDPC) method (Tiampo et al., 2002). We place our sensors in these "hotspots" to potentially improve the likelihood of proximal measurements of a large earthquake.

We have deployed four classes of sensor systems, the QF1005, QF-1003, QF-1000, and QF-HS, to extend the range of measurement opportunities. Design details are given in the next section. Ten, high-performance systems, the QF$1005 \mathrm{~s}$, perform detailed measurements of ULF magnetic activity. Positions of these ten systems are given in Table 1. The remaining fifty-eight sensor systems are lower cost systems that extend the geographic coverage of the network to increase our likelihood of measurements near a large earthquake, but with reduced measurement sensitivity at lower frequencies. Figure 1 shows the location of CalMagNet sensor systems.

Complementing the installed sites, we have a transportable QF-1005 for specialized field campaigns of short-term measurements (weeks to months). Similar in operational principle to the system described by Karakelian et al. (2000), this transportable unit will be installed near the epicenter of 
Table 1. Location summary of the ten QF-1005 sensor systems in geodetic coordinates with calculated L-shell values.

\begin{tabular}{cccccc}
\hline ID & Name & Lat. $\left({ }^{\circ} \mathrm{N}\right)$ & Lon. $\left({ }^{\circ} \mathrm{W}\right)$ & Elev. $(\mathrm{m})$ & L-Value \\
\hline HDW & Honeydew & 40.244 & 124.116 & 123 & 1.982 \\
HLD & Healdsburg & 38.694 & 122.947 & 85 & 1.895 \\
EMP & East Milpitas & 37.415 & 121.780 & 637 & 1.832 \\
PTV & Portola Valley & 37.336 & 122.196 & 457 & 1.822 \\
MET & Mettler & 35.055 & 119.031 & 136 & 1.731 \\
BEC & LeBec & 34.827 & 118.897 & 1324 & 1.721 \\
YUC & Yucaipa & 34.072 & 117.081 & 981 & 1.701 \\
CRN & Corona & 33.834 & 117.585 & 379 & 1.684 \\
OCT & Ocotillo Wells & 33.142 & 116.136 & 60 & 1.665 \\
JLN & Julian & 33.101 & 116.597 & 1280 & 1.658 \\
\hline
\end{tabular}

Table 2. Summary of CalMagNet sensor system characteristics.

\begin{tabular}{lcccc}
\hline \multirow{2}{*}{ Characteristic } & \multicolumn{4}{c}{ System Type } \\
& HS & 1000 & 1003 & 1005 \\
\hline Number of stations & 24 & 11 & 23 & 10 \\
Geophone & no & no & yes & yes \\
Extra Channels & 0 & 0 & 4 & 4 \\
Samples per second & 20 & 20 & 20 & 32 \\
Bits per sample & 12 & 12 & 16 & 24 \\
GPS Timing & no & no & yes & yes \\
Coil Specs. & & & & \\
Gain @ 1 Hz (V/pT) & $2 \times 10^{-3}$ & $2 \times 10^{-3}$ & $2 \times 10^{-3}$ & $10^{-3}$ \\
Noise @ 1 Hz (pT) & 3 & 3 & 3 & 0.1 \\
Pass Band (Hz) & $0.5-4$ & $0.5-4$ & $0.5-4$ & $0.001-12$ \\
\hline
\end{tabular}

future earthquakes to search for any post-earthquake ULF anomalies. It will also be used for specialized remote referencing measurements when local signals under study are dominated by larger, wide-area signals (Egbert, 1997; Larsen, 1989).

\section{Sensor systems}

CalMagNet is composed of four classes of sensor systems. Their characteristics are summarized in Table 2, including the number and types of systems, induction coil parameters, extra channels, sampling rate and resolution, and GPS timetagging. In the rest of this section, we provide a detailed summary of the ten high-performance stations, the QF-1005s, and include brief summaries of the additional three classes.

\subsection{QF-1005 System}

Figure 2 is a block diagram of the major components of the QF-1005. The primary instruments for the QF-1005 are three orthogonal induction coil magnetometers, the ANT/4, designed and manufactured by Zonge Engineering (Zonge, 2006). The transfer function and noise floor and gain of a typical ANT/4 coil are plotted in Fig. 3, panels a and b, respectively and summarized in Table 3 .
A high pass analog filter rolls off the gain below $0.25 \mathrm{~Hz}$ to suppress the monotonic increase in signal strength at lower frequencies. An anti-aliasing, analog filter rolls off the gain above $12 \mathrm{~Hz}$ to suppress $60 \mathrm{~Hz}$ noise while still allowing detection of the first Schumann resonance. During calibration, coil characteristics are optimized to achieve high coherence between separate coils, greater than $99 \%$. After field measurements during system testing, an additional filter was installed to further reduce $60 \mathrm{~Hz}$ noise contamination. An analog, 5-pole Butterworth filter with a $11.5 \mathrm{~Hz}$ cutoff frequency was installed to provide a total of $100 \mathrm{~dB}$ attenuation at $60 \mathrm{~Hz}$.

Three coils are installed at each site. The first coil is aligned in the geodetic north/south direction $\left(15^{\circ} \pm 2^{\circ}\right.$ west of geomagnetic north) with positive signal levels indicating a magnetic field vector pointing to the north.

The second coil is aligned in the geodetic east/west direction $\left(15^{\circ} \pm 2^{\circ}\right.$ south of geomagnetic east) with positive signal levels indicating a magnetic field vector pointing to the east. The third coil is installed vertically using a plumb line. A positive signal indicates a magnetic field vector pointing in the down direction.

A calibration signal is applied twice a day to each coil. At approximately local noon and midnight (within 5 min of the hour mark), an $8 \mathrm{nT}$ peak-to-peak signal at $1 \mathrm{~Hz}$ is applied to each coil through a calibration coil built into the magnetometers. The calibration signal lasts five minutes. The sensor response is monitored for any degradation in signal quality.

Analog data channels are digitized by a commercial 8-channel, 24-bit analog-to-digital converter system, the PAR $8 \mathrm{CH}$ by Symmetric Research. Samples are taken at a frequency of 32 samples per second. The sample rate is adjustable and was set to provide reasonable data file sizes for our frequency band of interest, less than $10 \mathrm{~Hz}$. It was also set such that any $60 \mathrm{~Hz}$ signal contamination would fold over onto $4 \mathrm{~Hz}$ during Fourier-based spectral analysis (versus $0 \mathrm{~Hz}$ when sampled at $30 \mathrm{~Hz}$ ). The PAR $8 \mathrm{CH}$ uses eight independent analog-to-digital converter chips, the ADS 1210 from Burr Brown, to reduce cross-talk noise contamination. 


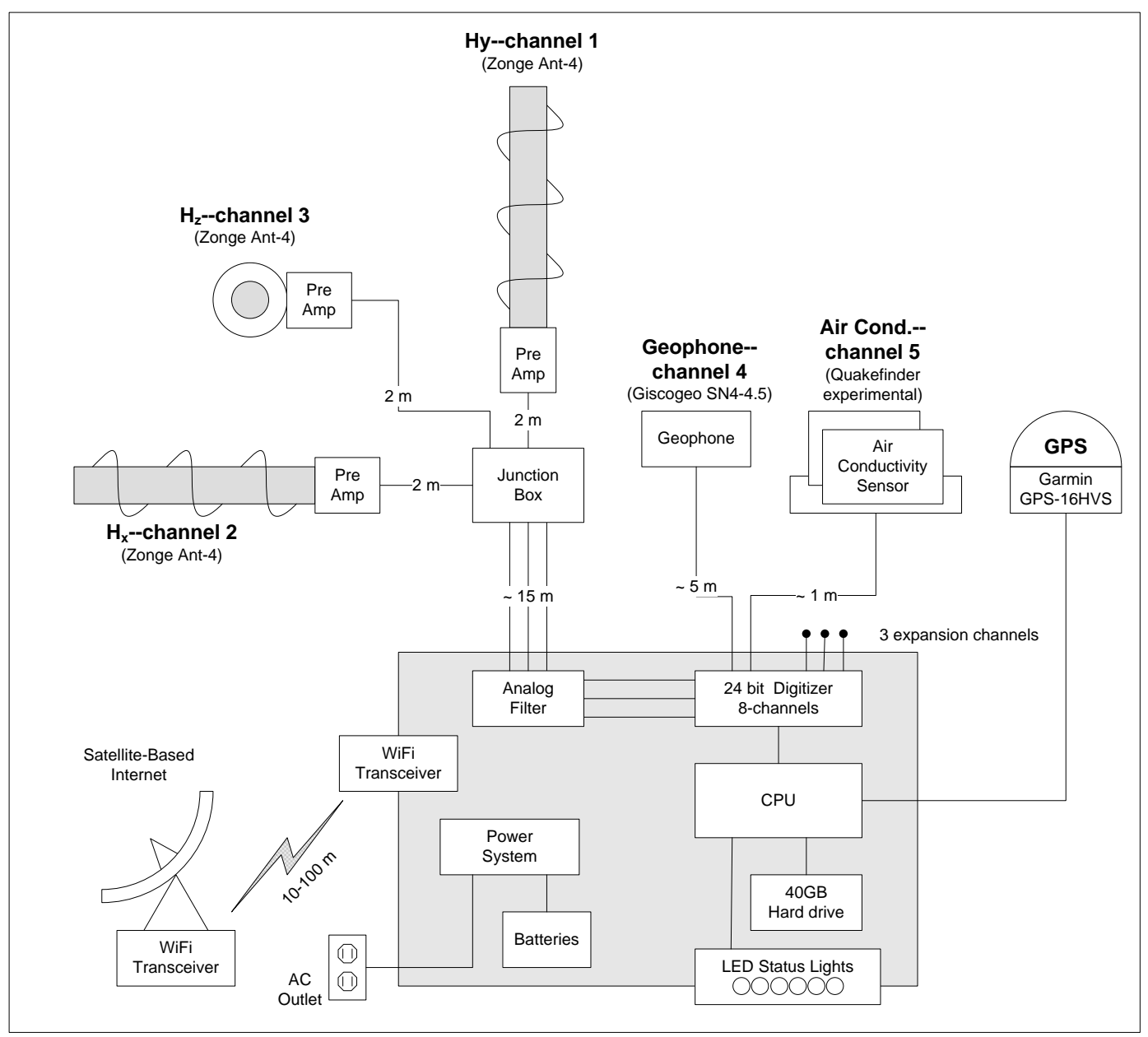

Fig. 2. Block diagram of QF-1005 system. Hx, Hy. and Hz represent the 3 search-coil magnetometers, oriented in the North, East and nadir directions respectively. A single axis geophone measures local motion. An experimental air conductivity sensor is attached, and there are three spare channels for future sensor expansions. The large gray central box houses analog filters, sampling systems, a computer, power systems, and communication equipment. Data transfers to the data center typically occur over satellite phone or Internet connections.

The primary CalMagNet instruments, namely the induction coil magnetometers, are susceptible to motion-induced noise. Oscillating microradian tilts of the induction coils in the ambient earth magnetic field result in signals that are similar to naturally occurring signals. Ideally, coils should be placed near broadband seismometer stations to provide detailed monitoring of ground motion (Karakelian et al., 2000). Due to cost constraints, CalMagNet systems are not all located near broadband seismometer stations. To augment the existing wide-area seismic monitoring stations, we have installed secondary sensors on the systems, a Giscogeo geophone SN 4-4.5, that are five times more sensitive than the induction coils to motion at frequencies greater than $4 \mathrm{~Hz}$.

Four of the eight channels of the data acquisition are available for experimental and future sensor expansion. Currently, at several sites, experimental air conductivity sensors are installed. A number of experimental dipole electric antennas from Quasar Federal Systems (Delory et al., 2005) will be deployed in the near future to test their operation.

Each system is controlled by an embedded PC-104 processor system that manages data acquisition and transfer. Raw sample data from the sensors is stored in five minute blocks, and archived locally on a standard 2.5 inch hard drive with a capacity greater than $40 \mathrm{~GB}$. This provides over 600 days of storage of 8 channels sampled at $32 \mathrm{~Hz}$ and 24 bits for local archiving during the presence of communication failures.

Raw data collected over a $24 \mathrm{~h}$ period is transferred nightly to a data center over commercial, satellite-based Internet links. The data center archives sensor data, monitors health of sensor systems, and produces a variety of products such as daily dynamic spectrograms (Cutler, 2005). Future upgrades will stream the data in near real-time to the data center for more timely processing. 

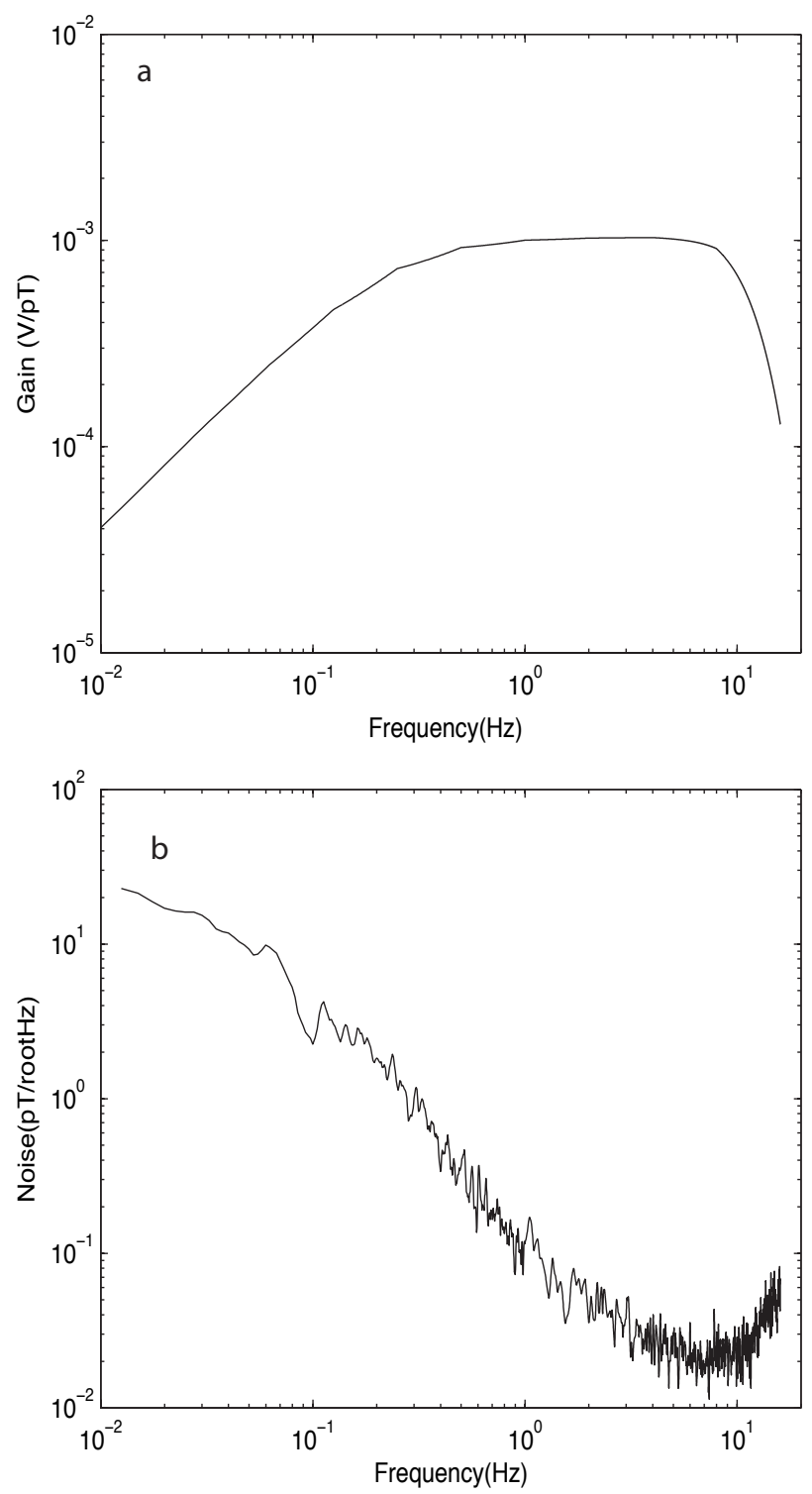

Fig. 3. Characteristics of a single ANT/4 magnetometer. (a) Plot of gain versus frequency for the ANT/4, pre-amplifier, and filter. (b) Noise floor of the ANT/4 magnetometer. These are measured values from coils deployed in CalMagNet. They were obtained by taking two coils to a remote, low-noise location, and differencing the signals to remove common mode signal while leaving only the internal noise of the system. Values differ slightly from Table 3 due to local environmental noise conditions during this particular test.

GPS-based time tagging of data samples is provided by a Garmin GPS-16 HVS receiver. The pulse per second output of the receiver (accurate to \pm 1 microsecond) is combined with a 800 nanosecond counter on the PAR $8 \mathrm{CH}$ to provide an overall timing error less than 3.3 microseconds.
Table 3. Summary of typical Zonge ANT/4 magnetometer gain and noise characteristics. Values are average measurements made from multiple ANT/4 tests in quiet locations.

\begin{tabular}{ccc}
\hline Frequency $(\mathrm{Hz})$ & Gain $(\mathrm{V} / \mathrm{pT})$ & Noise $\left(\mathrm{pT} / \mathrm{Hz}^{\frac{1}{2}}\right)$ \\
\hline 0.001 & $3 \times 10^{-6}$ & 250 \\
0.01 & $3 \times 10^{-5}$ & 15 \\
0.1 & $3 \times 10^{-4}$ & 1.5 \\
1 & $10^{-3}$ & 0.1 \\
10 & $10^{-3}$ & 0.02 \\
\hline
\end{tabular}

\subsection{Additional sensor systems}

In 1998, the initial CalMagNet sites, QF-HS systems, were deployed through an educational program where high school students built low-cost, "Heathkit-like" systems. The educational program taught students about scientific instrumentation and geomagnetic studies.

In 2001, we began upgrading to commercial versions, increasing both reliability and sensitivity. These are the QF1000 systems. In 2003, NASA provided funding to build and deploy twenty new sensors in the Mojave Desert of southern California. Through this NASA contract, our upgraded sensor system, the QF-1003, now includes GPS time synchronization, a Globalstar communication system, an air conductivity sensor, and a geophone. The third generation system, the QF-1005, has been described above.

\section{Example signals and analysis}

Survey and classification of ULF signal sources received by the CalMagNet is underway in our data center (Cutler, 2005). Typical signals include geomagnetic pulsations resulting from ionospheric and magnetospheric processes (Jacobs, 1970), cultural noise such as public transportation systems (Liu, 1999), and movement of the coil in the earth's magnetic field (Karakelian et al., 2000). In this section, we provide an overview of typical signals received by CalMagNet and example analysis efforts.

\subsection{Geomagnetic pulsations}

Pc 1 geomagnetic pulsations are typical signals received by the network (Jacobs, 1970).

These waves are thought to originate in the equatorial region of the outer magnetosphere (Cornwall, 1965), propagate along field lines into the high latitude ionosphere, and propagate down to low latitudes within the F2-region ionospheric duct (Fraser, 1968; Manchester, 1968), where they are observed by our instruments.

Figure 4 shows Pc 1 geomagnetic micropulsations received by the network. Data from 17-18 April 2006 is plotted from two sites, East Milpitas near San Francisco and 

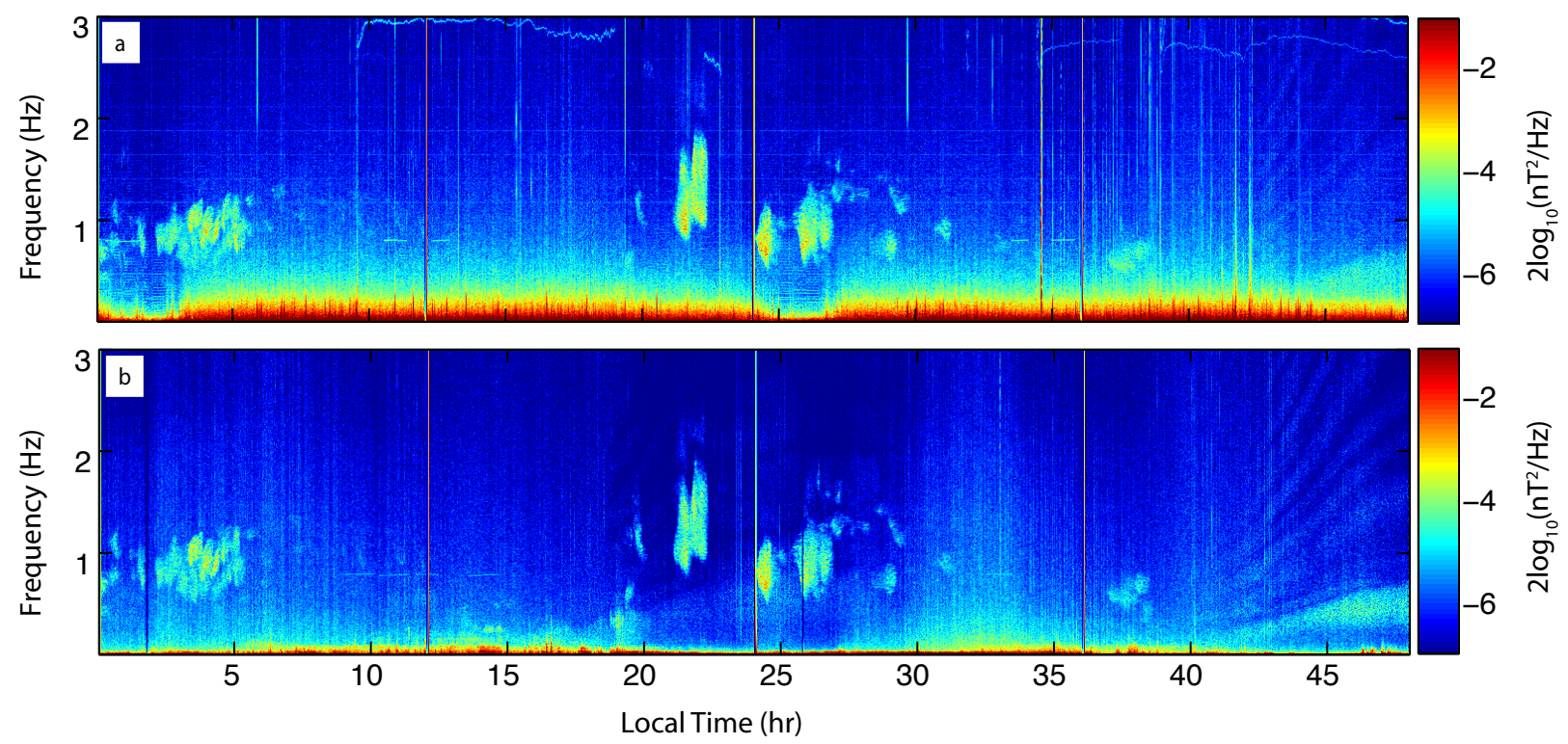

Fig. 4. Dynamic spectrograms on 17-18 April 2006 at two CalMagNet Sites: (a) East Milpitas (EMP), near San Francisco, and (b) Julian (JLN), near San Diego. Strong Pc 1 pulsations occur at both sites between 0.5 and $2 \mathrm{~Hz}$ periode.

Julian near San Diego. Multiple Pc1 pulsations are seen during the early morning and night at both sites due to a strong magnetic storm that occurred 14 April 2006.

\subsection{Ionospheric Alfvén Resonator}

Another common ULF signal is the spectral resonant structure (SRS) of the the ionospheric Alfvén resonator (IAR) (Bosinger et al., 2002). According to current models, the primary excitation source is electromagnetic emissions from global thunderstorm activity (Belyaev et al., 1990). Characteristics of the IAR such as the fundamental frequency are governed by local ionospheric conditions. Distributed measurements by CalMagNet of SRS properties allow measurement of ionospheric properties over California.

Wave parameters of typical SRS activity as measured by CalMagNet are shown in Fig. 5. These parameters are calculated following the methodology of Means (1972) and Fowler et al. (1967) using the wave detection and characterization approach outlined by Bortnik et al. (2007).

Plot a shows the zenith angle of three days of data from site JLN starting from noon local time on 03 May 2006. Plots b-f plot additional wave parameters from a focused time period, $21 \mathrm{~h}$ of data starting at 1400 local time on 05 May 2006 to 0700 on 06 May 2006. Plot $g$ is the power spectral density of combined three channel data from three CalMagNet sites.

Several interesting characteristics of SRS are highlighted in this example.

First, the recently described fine structure of the IAR (Bosinger et al., 2004) is clearly seen in plots b-e.
Second, plot $g$ shows the simultaneous wide-area occurrence and local characteristics of SRS. SRS is measured simultaneously at sites HLD, MET, and JUL, which are are separated by over $840 \mathrm{~km}$. The variations in center frequency and signal strength at each of the sites shows the differences in local ionospheric conditions.

The spatial dimensions of the ionosphere that are characterized by a single site's measurement of SRS are yet to be determined.

\subsection{Response to ground motion}

The responsive of the system to ground motion is shown in Fig. 6. On 25 March 2006 at 17:56 PST, a $M_{w} 4.6$ offshore earthquake occurred $25 \mathrm{~km}$ to the northwest of station Honeydew. Figure 6 plots the geophone and vertical induction coil data from the site. The arrival of the $\mathrm{P}$ and $\mathrm{S}$ waves are clearly seen in both channels. As shown, the geophone is an indicator for noise contamination of the induction coils by motion.

\subsection{Cultural noise}

Cultural noise sources, such at the commuter trains in the San Francisco Bay Area, have the potential to contaminate CalMagNet systems (Liu, 1999). The effects of narrow band noise sources such as power lines can be reduced through analog and digital filtering techniques. Broadband, non deterministic noise (such as nearby automotive traffic, windinduced motion through tree root systems, farming machinery, and moving ferromagnetic materials such as chain link fences) must be characterized in the long term and specific 

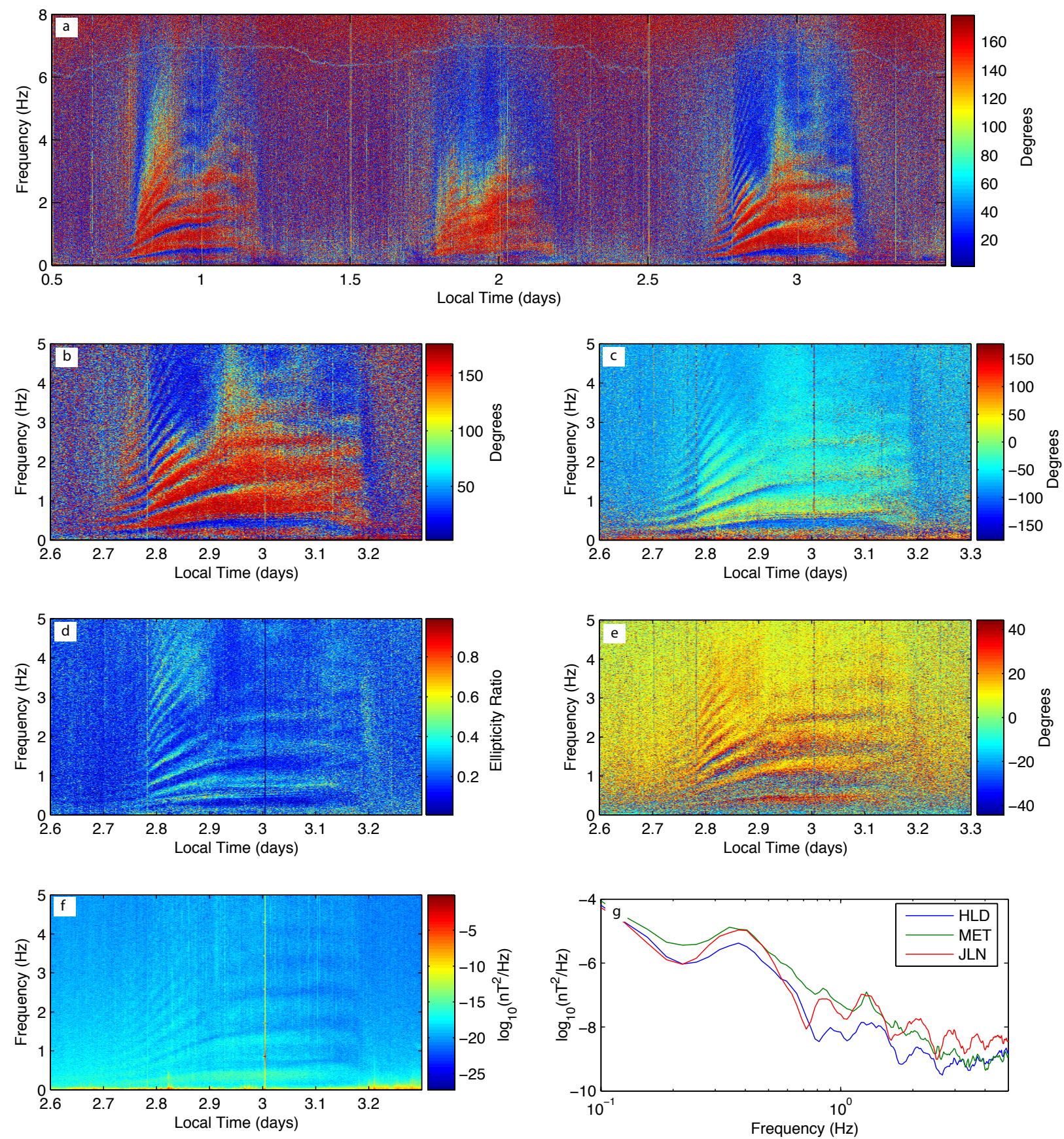

Fig. 5. Typical SRS as measured by CalMagNet. (a) Zenith angle of k-vector with respect to the z-axis. Three days of data are shown, starting at noon local time on 03 May 2006 from site JLN-605. (b) Zenith angle, zoomed in view of the third SRS event. (c) Azimuth angle of k-vector with respect to the x-axis. (d) Ellipticity. (e) Angle of major ellipse axis with respect to the x-axis. (f) Power of combined three-axis signal. (g) Power spectral density at three CalMagNet sites averaged over 1-3 am local time on 06 May 2006 . The vertical bars at time $1,1.5,2,2.5,3$, and 3.5 are the calibration signals.

noise characteristics cataloged. These sources are site dependent and libraries of local noise examples are under development. In extreme cases of noise contamination, sites can be moved to quieter locations.
Low frequency noise from the rapid transit system in the San Francisco area, BART, is seen in Fig. 4. In the top plot, broadband noise below $0.5 \mathrm{~Hz}$ is seen and corresponds to BART train activity. There is a noticeable two hour decrease after midnight which corresponds to the typical reduction in 

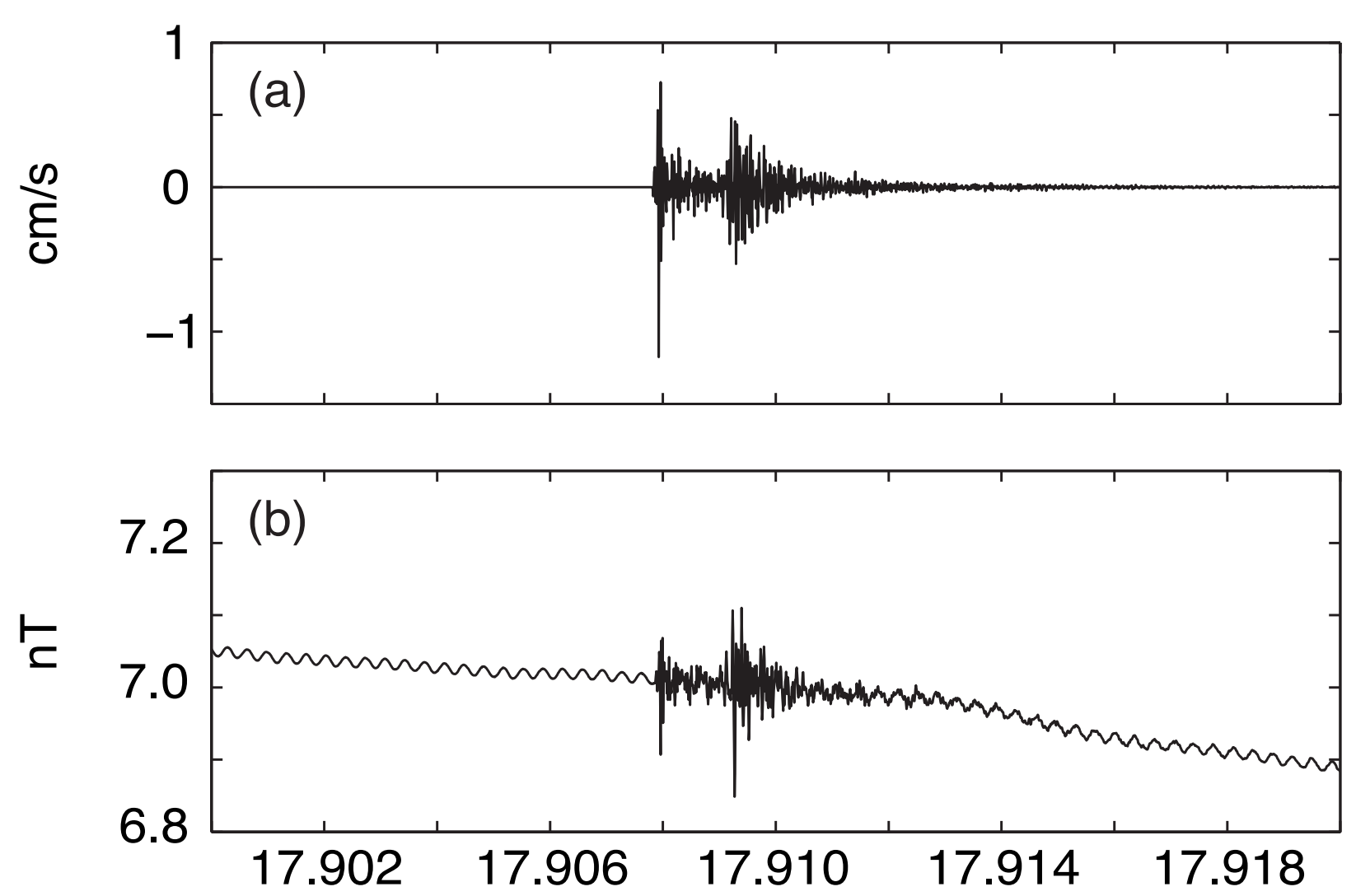

Local Time (hr)

Fig. 6. Data during a nearby earthquake is plotted from station HDW, Honeydew. (a) normalized geophone data. (b) normalized data from the vertical induction coil. The low-amplitude periodic signal in (b) is a nearby cultural noise source that appears during the day. Time is given in local time as the number of hours since midnight, 25 March 2006. System response to the P and S waves are clearly seen in both plots. See Karakelian et al. (2002) for additional coseismic signals.

BART traffic. Spurious harmonic tones between $1-2 \mathrm{~Hz}$ and a wandering tone near $3 \mathrm{~Hz}$ are also visible.

\section{Conclusions}

We have deployed an array of sixty-eight ULF monitoring stations in California called the CalMagNet. Frequencies from $1 \mathrm{mHz}$ to $12 \mathrm{~Hz}$ are measured at ten high-performance stations. Frequencies from $0.5 \mathrm{~Hz}$ to $4 \mathrm{~Hz}$ are measured with fifty-eight lower-cost stations. The purpose of the network is to provide detailed, multi-point measurements of ULF magnetic fluctuations such as geomagnetic micropulsations and to monitor ULF activity in active fault zones for any potential earthquake related signals.

A data center is currently under development to support CalMagNet sensors (Cutler, 2005). Data is archived on Quakefinder servers, and external access to raw data is pro- vided to partnered researchers. A variety of daily data products are produced including dynamic spectrograms, transfer function compensated time series, and magnetic activity indices that summarize power levels in distinct frequency bands. Several examples of our data have been presented in the present paper, and work is currently underway to make such information available for public viewing online.

Acknowledgements. The authors wish to thank our generous supporters, especially Celeste Ford and Stellar Solutions. This work was conducted in part by NASA cooperative agreement NNG04GD16A. Many thanks to B. Camins and B. Hunter for all the sweat and blisters it took to install the CalMagNet sites. We thank S. Klemperer and T. Fraser-Smith for their feedback and comments. We also thank our reviewers and editors.

Edited by: M. Contadakis

Reviewed by: S. Klemperer and another anonymous referee 


\section{References}

Armadillo, E., Bozzo, E., Cerv, V., Santis, A. D., Mauro, D. D., Gambetta, M., Meloni, A., Pek, J., Speranza, F., and Schultz, A.: Geomagnetic depth sounding in the northern apennines (Italy), Earth, Planets and Space, 53, 385-396, 2001.

Bakun, W. H. and Lindh, A. G.: The Parkfield, California, earthquake prediction experiment, Science, 229, 619-624, 1985.

Belyaev, P., Polyakov, S., Rapoport, V., and Trakhtengerts, V. Y.: The ionospheric Alfvén resonator, J. Atmos. Sol.-Terr. Phy., 52:9, 781-788, 1990.

Bernardi, A., Fraser-Smith, A. C., McGill, P. R., and Villard, O. G. J.: ULF magnetic field measurements near the epicenter of the Ms 7.1 Loma Prieta earthquake, Phys. Earth Planet. In., 68, 45-63, 1991.

Bijoor, S., Glen, J., McPhee, D. K., and Klemperer, S. L.: Ultra-low frequency electromagnetic monitoring of earthquakes in the San Francisco Bay Area: Initial results of an Earthscope PBO project, EOS Transactions AGU, Fall Meeting, T51B-1343, 2005.

Bortnik, J., Cutler, J. W., Dunson, C., and Bleier, T. E.: An automatic wave detection algorithm applied to Pc 1 pulsations, J. Geophys. Res., 112, A04204, doi:10.1029/2006JA011900, 2007.

Bosinger, T., Haldoupis, C., Belyaev, P. P., Yakunin, M. N., Semenova, N. V., Demekhov, A. G., and Angelopoulos, V.: Spectral properties of the ionospheric Alfvén resonator observed at a low-latitude station (L=1.3), J. Geophys. Res., 107(A10), 1281, doi:1029/2001JA005076, 2002.

Bosinger, T., Demekhov, A., and Trakhtengerts, V.: Fine structure in ionospheric Alfvén resonator spectra observed at low latitude ( $\mathrm{L}=1.3)$, Geophys. Res. Lett., 31, L18802, doi:10.1029/2004GL020777, 2004.

Campbell, W. H.: Introduction to Geomagnetic Fields, 2nd Edition, Cambridge University Press, United Kingdom, 2003.

Cornwall, J. M.: Cyclotron instabilities and electromagnetic emission in the ultra low frequency and very low frequency ranges, $\mathrm{J}$. Geophys. Res., 70(1), 61-69, 1965.

Cutler, J.: A data fusion center for seismo-electromangetic community, The 3rd Annual Meeting of GEON, San Diego, CA, 2005.

Delory, G. T., Grimm, R. E., Nielsen, T., and Farrell, W. M.: Detection of subsurface liquid water using magnetotellurics on Mars, EOS Transactions AGU, Fall Meeting, 86(52), P31C0217, 2005.

Egbert, G. D.: Robust multiple-station magnetotelluric data processing, Geophys. J Internat., 130 pp., 475-496, 1997.

Fowler, R., Kotick, B., and Elliott, R.: Polarization analysis of natural and artificially induced geomagnetic pulsations, J. Geophys. Res., 72, 2871-2883, 1967.

Fraser, B. J.: Temporal variations in Pc 1 geomagnetic micropulsations, Planet. Space Sci., 16, 111, 1968.

Fraser-Smith, A. C., Bernardi, A., McGill, P. R., Ladd, M. E., Helliwell, R. A., and O. G. Villard, J.: Low-frequency magnetic field measurements near the epicenter of the Ms 7.1 Loma Prieta earthquake, Geophys. Res. Lett., 17, 1465-1468, 1990.

Gregori, G. P. and Lanzerotti, L. J.: Geomagnetic depth sounding by induction arrow representation: A review, Rev. Geophys. Space Phys., 18, 203-209, 1980.

Hayakawa, M.: Atmospheric and Ionospheric Electromagnetic Phenomena Associated with Earthquakes, Terrapub, Tokyo, 996, 1999.

Hebden, S. R., Robinson, T. R., Wright, D. M., Yeoman, T., Raita,
T., and Bosinger, T.: A quantitative analysis of the diurnal evolution of ionospheric Alfvén resonator magnetic resonance features and calculation of changing IAR parameters, Ann. Geophys., 23, 1711-1721, 2005, http://www.ann-geophys.net/23/1711/2005/.

Jacobs, J. A.: Geomagnetic Micropulsations, Springer-Verlag, 1970.

Johnston, M. J. S.: Review of electric and magnetic fields accompanying seismic and volcanic activity, Surv. Geophys., 18, 441476, 1997.

Karakelian, D., Klemperer, S. L., Fraser-Smith, A. C., and Beroza, G. C.: A transportable system for monitoring ultra low frequency electromagnetic signals associated with earthquakes, Seismol. Res. Lett., 71, 423-436, 2000.

Karakelian, D., Beroza, G. C., Klemperer, S. L., and FraserSmith, A. C.: Analysis of ultra-low frequency electromagnetic field measurements associated with the 1999 M 7.1 Hector Mine earthquake sequence, Bot. Bull. Acad. Sinica, 92, 1513-1524, 2002.

Larsen, J. C.: Transfer functions: smooth robust estimates by leastsquares and remote reference methods, Geophys. J. Internat., 99, 645-663, 1989.

Liu, T. T.: Ultra-low frequency magnetic fields in the San Francisco Bay Area: Measurements, models, and signal processing, Ph. D. thesis, Stanford University, June 1999.

Manchester, R. N.: Correlation of Pc 1 Micropulsations at Spaced Stations, J. Geophys. Res., 73(11), 3549-3556, 1968.

Mann, I. R., Milling, D. K., Kale, A., Rae, I. J., Dent, Z. C., Loto'Aniu, T., and Wallis, D. D.: The expanded and upgraded CANOPUS magnetometer array: An extensive ground-based magnetometer array in the THEMIS and ILWS era, American Geophys. Union, Spring Meeting, SM31A-02, May 2004.

Means, J.: Use of the three-dimensional covariance matrix in analyzing the polarization properties of plane waves, J. Geophys. Res., 77, 5551-5559, 1972.

Boyd, O. S., Morrison, H. F., Fraser-Smith, A., and Park, S.: An array for monitoring ULF fields and changes in resistivity, The 13th Workshop on Electromagnetic Induction in the Earth, The International Association of Geomagnetism and Aeronomy, Onuma, Japan, 12-18 July 1996.

Neal, S. L., Mackie, R. L., Larsen, J. C., and Schultz, A.: Variations in the electrical conductivity of the upper mantle beneath North America and the Pacific Ocean, J. Geophys. Res., 106(B4), 8229-8242, 2000.

Park, S. K., Johnston, M. J. S., Madden, T. R., Morgan, F. D., and Morrison, H. F.: Electromagnetic precursors to earthquakes in the ULF band: a review of observations and mechanisms, Rev. Geophys., 31, 117-132, 1993.

Park, S. K., Dalrymple, W., and Larsen, J. C.: The 2004 Parkfield earthquake: Test of the electromagnetic precursor hypothesis, J. Geophys. Res., 112, B05302, doi:10.1029/2005JB004196, 2007.

Roeloffs, E. and Langbein, J.: The earthquake prediction experiment at Parkfield, Rev. Geophys., 32, 315-336, 1994.

Rostoker, G., Samson, J. C., Creutzberg, R., Hughes, T. J., Mc Diarmid, D. R., McNamar, A. G., Jones, A. V., Wallis, D. D., and Cogger, L. L: CANOPUS - a ground-based instrument array for remote sensing the high latitude ionosphere during the ISTP/GGS program, Space Sci. Rev., 71, 743-760, 1995.

Schmucker, U.: Geophysics of the Solid Earth, the Moon and the 
Planets, 2, Springer, 1985.

Simpson, F. and Bahr, K.: Practical Magnetotellurics, Cambridge, 2005.

Tiampo, K. F., Rundle, J. B., McGinnis, S., Gross, S. J., and Klein, W.: Mean field threshold systems and phase dynamics: An application to earthquake fault systems, Europhys. Lett., 60, 481-487, 2002.
Zonge: Zonge engineering and research organization. http://www. zonge.com/,last access: 15 January 2008, 2006. 